\title{
Marcadores moleculares como herramientas en la identificación y análisis genético de especies vectores de interés en salud pública, género Lutzomyia (Grupo Verrucarum Theodor, 1965)
}

\begin{abstract}
Molecular markers as tools in the identification and genetic analysis of vector species of interest in public health, type Lutzomyia (Verrucarum Theodor group, 1965)
\end{abstract}

Carol Yovanna Rosero-Galindo', Franco Andrés Montenegro-Coral2, Juan Pablo García-López³

1 Bióloga. PhD en Ciencias - Biología. Grupo Interdisciplinario de Investigación en Salud-Enfermedad (GIISE). Profesora investigadora, Facultad de Medicina, Universidad Cooperativa de Colombia. Pasto, Colombia. e-mail: carol.roserog@campusucc.edu.co

2 Biólogo. Especialista en Ecología, Especialista en Planeación Ambiental y Manejo de Recursos Naturales. Profesor Investigador, Facultad de Medicina, Universidad Cooperativa de Colombia. Pasto, Colombia. e-mail: franco.montenegro@campusucc.edu.co

3 Biólogo. Docente - Investigador. Departamento de Biología. Universidad de Nariño. Pasto, Colombia. e-mail: jgarcia@biologia.udenar.edu.co

Rosero-Galindo CY, Montenegro-Coral FA, García-López JP. Marcadores moleculares como herramientas en la identificación y análisis genético de especies vectores de interés en salud pública, género Lutzomyia (Grupo Verrucarum Theodor, 1965). Univ. Salud. 2016;18(1):138-155. DOI: http://dx.doi.org/10.22267/rus.161801.26

\begin{abstract}
Resumen
Introducción: Los insectos flebotomíneos del grupo Verrucarum, son vectores de interés en salud pública dada su participación en la transmisión de las enfermedades tropicales: leishmaniasis y bartonelosis. El empleo de marcadores moleculares como herramienta de identificación y análisis genético de especies que se comportan como vectores potenciales y confirmados, muestran un uso ampliamente difundido, que genera un conjunto de información que se actualiza constantemente, más aun cuando la identificación de estas especies, es prioritaria en los países donde estas enfermedades ocurren de forma recurrente. Objetivo: Se presenta una revisión sistemática con el objetivo de recopilar y presentar una actualización sobre el uso de marcadores moleculares empleados en especies del grupo Verrucarum. Materiales y métodos: Esta búsqueda se efectuó en un rango de literatura relevante publicada en un periodo de 24 años que incluyó 23 artículos en la temática de genética y biología molecular de flebótomos y 39 artículos de otras áreas como sistemática, ecología de flebótomos y microbiología de agentes patógenos asociados. Resultados: Los resultados muestran el empleo de los genes que codifica para el ARN ribosomal 18S y para el citocromo oxidasa subunidad I como los más efectivos para observar las relaciones filogenéticas entre especies del grupo Verrucarum; el uso del gen citocromo b como carácter taxonómico alternativo para identificar correctamente los flebótomos y, el uso de secuencias espaciadoras de la transcripción interna del ARN ribosomal y el gen citocromo b para determinar la variación genética intra e interespecífica de las poblaciones. Conclusión: Esta revisión contribuirá a estudios filogenéticos de vectores de importancia en salud pública.
\end{abstract}

Palabras clave: Vectores de enfermedades, marcadores genéticos, genética de población, filogeografía; enfermedades tropicales; Lutzomyia. (Fuente: DeCS, Bireme). 


\begin{abstract}
Introduction: The phlebotomine flies of the Verrucarum group are vectors of interest in public health because of their participation in the transmission of the tropical diseases: leishmaniasis and bartonellosis. The use of molecular markers, as a tool for the identification and genetic analysis of species that behave as potential and confirmed vectors, show a widely disseminated use that generates a set of information that is constantly updated, even more when the identification of these species is a priority in the countries where these diseases occur on a recurring basis. Objective: The paper presents a systematic review that aims at compiling and presenting an update on the use of molecular markers employed in species of the Verrucarum group. Materials and methods: This search was conducted in a range of relevant literature published in a period of 24 years which included 23 articles on the subject of genetics and molecular biology of sand flies, and 39 articles from other areas such as systematic, ecology of sand flies and microbiology of related pathogenic agents. Results: The results show the use of genes coding for ribosomal 18S RNA and cytochrome oxidase subunit I as the most effective to observe the phylogenetic relationships among species of the Verrucarum group; the use of the cytochrome b gene as alternative taxonomic character to correctly identify the sand flies, and the use of spacer sequences of the internal transcript of the ribosomal RNA and the Cytochrome b gene to determine the genetic variation of intra- and inter-specific populations. Conclusions: This review will contribute to phylogenetic studies of vectors of public health importance.
\end{abstract}

Keywords: Vectors of diseases, genetic markers, population genetics, phylo-geography; tropical diseases; Lutzomyia. (Source: DeCS, Bireme).

\section{Introducción}

Los flebotomíneos son pequeños dípteros hematófagos de la familia Psychodidae (Newman, 1834), subfamilia Phlebotiminae (Rondani, 1840) con siete géneros reconocidos; dos de ellos, Phlebotumus y Lutzomyia, responsables de la transmisión de protozoos del género Leishmania (Ross, 1903) y de la bacteria Bartonella bacilliformis (Strong et al., 1913), Strong et al., 1915, causantes de leishmaniasis y bartonelosis en humanos.1-4

Dentro del género Lutzomyia, se incluye al grupo Verrucarum Theodor 5 1965, el cual presenta como caracteres diagnósticos más importantes: Fémur posterior sin espinas, ascoides antenales sin largas proyecciones posteriores y quinto segmento palpal de mayor longitud al tercero.6 En ambos sexos el palpómero $\mathrm{V}$ es mayor que el palpómero III, observándose en este último las espinas de Newstead. Los machos presentan antenas con ascoides cortos, un ápice que generalmente llega hasta la mitad del segmento, un ascoide en el flagelómero AXV, ausencia de sensilas en las rosetas de AXII al AXVI, los dististilos con 3 o 4 espinas fuertes y una cerda espiniforme preapical, los basistilos con o sin tufo de cerda, y los lóbulos laterales con un ápice de forma redondeada y con parámetros simples.7
Las hembras presentan ascoides cuyo ápice pueden llegar hasta la región subapical del segmento, hipofaringe con dientes, un cibario con 4 dientes horizontales (los verticales dispuestos en 1 ó 2 hileras transversales) y un arco esclerosado completo, las espermatecas con un ducto común largo, el cuerpo predominantemente vesiculoso con estriación transversal, con o sin anillo apical y una cabeza visible.7

El grupo Verrucarum se encuentra constituido por cuatro series: verrucarum (Townsend, 1913), serrana (Damasceno y Arouck, 1949), townsendi (Ortiz, 1959), y pia (Fairchild y Hertig, 1961) . Las series se conforman con base en la similitud morfológica de caracteres asociados a la genitalia del macho (espinas subterminales y tufo de setas):5,8-10

Los caracteres diagnósticos característicos para cada una de las series del grupo son: La serie verrucarum presenta cuatro espinas sobre el estilo, con dos espinas mediales pareadas y dos distales.5,12 La serie serrana, se caracteriza por presentar tres espinas.5 La serie townsendi $i_{12}$ presenta tres espinas distales y una espina basal aislada. Las hembras son isomórficas, y presentan faringe sin espinas posteriores, mientras que los machos presentan seta subterminal y un parámero no ramificado y en la serie pía los 
machos se caracterizan por presentar cinco espinas estilares, mientras las hembras tienen todas numerosos dientes laterales puntiagudos afilados en ambos lados de los dientes horizontales. 6,13,15

Las cuatro series del grupo incluyen 40 especies, de las cuales 10 son de importancia médica en Colombia.11 Serie verrucarum: Lutzomyia columbiana (Ristorcelli y Van Ty, 1941), Lutzomyia evansi (Nunez-Tovar, 1924), Lutzomyia nuneztovari (Ortiz 1954), Lutzomyia ovallesi (Ortiz 1952) y serie townsendi: Lutzomyia longiflocosa (Osorno, Morales, Osorno y Munoz, 1970), Lutzomyia quasitowsendi (Osorno, Osorno y Morales, 1972), Lutzomyia spinicrassa (Morales, Osorno, Osorno y Munoz, 1969), Lutzomyia torvida (Young, Morales y Ferro, 1994), Lutzomyia townsendi (Ortíz 1959), Lutzomyia youngi (Feliciangeli y Murillo, 1987).

En la transmisión de enfermedades parasitarias tropicales el estado vectorial de las especies clasificado como potencial y confirmado relaciona la bartonelosis con L. columbiana, leishmaniasis cutánea con $L$. columbiana, $L$. nuneztovari, $L$. ovallesi, L. quasitowsendi, L. spinicrassa, L. torvida, L. townsendi y L. youngi; leishmaniasis cutánea y mucocutánea con L. nuneztovari, L. longiflocosa, $L$. ovallesi, L. spinicrassa, L. torvida, L. townsendi y $L$. youngi; y/o leishmaniasis visceral con L. evansi.11

En este contexto, uno de los principales problemas en la epidemiología de una enfermedad parasitaria es la identificación taxonómica de la especie del vector, con el propósito de desarrollar programas eficaces de control, incriminación y conocimiento de la tasa de infección natural por especie.16

En el caso de la caracterización de los ejemplares del género Lutzomyia 17 (Grupo Verrucarum) se basa principalmente, en las diferencias que presentan algunas estructuras ubicadas en la cabeza, el tórax y el abdomen del imago, además de la quetotáxia, estudios de morfometría geométrica basada en estados de carácter morfológico, el sistema espiracular larval, la estructura coriónica y morfología del atrio genital, las cuales se aceptan como caracteres de clasificación del taxón en subgéneros, grupos, series, especies y subespecies.6,18-30

La clasificación de la subfamilia Phlebotominae es controversial, debido a que para la mayoría del elenco de especies que la conforman se conoce a uno de los dos sexos y se desconoce los estadios inmaduros (larvas y pupas), de esta manera, la determinación taxonómica de las especies flebotomíneas utiliza generalmente caracteres morfológicos del adulto.31,32 Sin embargo, ésta no es suficiente para diferenciar la totalidad de especies en este grupo de insectos.

De igual forma, la taxonomía de los flebotomíneos presenta algunas limitaciones, en el caso del género Lutzomyia, la presencia de especies hermanas o complejos de especies, como las especies que integran el grupo Verrucarum. Por otra parte, algunos taxones agrupan especies isomórficas e isométricas como el reportado para la serie townsendi $i_{33}$ integrada por un complejo de ocho especies crípticas entre ellas $L$. spinicrassa, $L$. towsendi y L. youngi con hembras isomorfas 33 y presencia de simpatría dentro de su distribución neotropical dificulta considerablemente la identificación morfológica al aplicar únicamente la morfología externa comparada,34-36 sumado a esto, en algunos casos las descripciones propuestas en la bibliografía puede ser insuficientes para algunos grupos de especies.37

Ante estas limitaciones, las aproximaciones moleculares son de gran utilidad en el caso de los insectos $\mathrm{y}$ en particular de aquellos de importancia médica, como los flebotomíneos, dado el hecho que las moléculas apoyan la identificación de especímenes en cualquiera de sus estados, e incluso de partes de ellos.38,39

De esta manera, se han identificado marcadores moleculares útiles en caracterización taxonómica de las especies del género Lutzomyia (Grupo Verrucarum). Es así, como los primeros estudios de taxonomía molecular se realizaron con marcadores proteicos y posteriormente con aplicaciones y modificaciones de la Reacción en Cadena de la Polimerasa (PCR), tales como, el análisis de polimorfismos en la longitud de los fragmentos de restricción (RFLPs), análisis de los 
fragmentos de longitud polimórfica amplificados al azar (RAPDs), amplificación de fragmentos de longitud polimórfica (AFLP), elementos de número variable repetidos en bloque (VNTR, y microsatélites ó STR) y polimorfismo de conformación de cadena sencilla PCR (SSCP-PCR).

Recientemente las investigaciones se han enfocado en la secuenciación de genes mitocondriales, entre los cuales se destacan los genes citocromo b (Cyt b) y la NADH deshidrogenasa Subunidad 4 (ND4) como los marcadores más empleados en el análisis genético de poblaciones y en estudios filogenéticos de especies de Lutzomyia.40-44 Por lo anterior, el objetivo de esta revisión es el de determinar el uso de los principales marcadores moleculares aplicados en la identificación molecular de especies del grupo Verrucarum, así como en el análisis de la diversidad genética de éste grupo.

\section{Materiales y métodos}

Un total de 62 artículos fueron revisados, de los cuales 15 fueron incluidos para la construcción de la tabla 1, porque referenciaron el uso de marcadores moleculares en al menos una especie del grupo Verrucarum. Se realizó una revisión descriptiva, para la localización de los documentos bibliográficos primarios se utilizaron varias fuentes documentales. La búsqueda bibliográfica automatizada fue efectuada en los meses de julio a agosto de 2014 en la base de datos Science Direct (http://www.sciencedirect.com/) y Pubmed del NCBI(http://www.ncbi.nlm.nih.gov/pubmed), y los buscadores académicos: Google Scholar (http://scholar.google.com.co/) y Google, con las extensión filetype:pdf, la búsqueda se realizó con el filtro de relevancia, extendiéndose a las dos primeras páginas de resultados encontrados, utilizando los descriptores: grupo Verrucarum, relaciones genéticas, estructura genética, relaciones sistemáticas, relaciones filogeográficas, relaciones filogenéticas, y primeras identificaciones, escritos en idioma español e inglés.

Los registros obtenidos fueron superiores a 10 tras la combinación de las diferentes palabras clave, representados en 62 referencias bibliográficas, empleando como filtro de escogencia un rango de 24 años a partir de la fecha de búsqueda (1990 - 2014), adicionalmente se tuvo en cuenta un solo artículo del año 1942 dada la importancia de la temática tratada. Para evitar contradicciones en la literatura, se tuvo como criterio de selección la referencia más actual del punto de vista en discusión.

\section{Resultados y discusión}

El análisis de la información permitió la organización de los resultados en la tabla 1, presentando una actualización del uso de los marcadores moleculares en especies del género Lutzomyia; en algunos casos fue necesario especificar la serie del grupo Verrucarum al que pertenecen las especies. Se observó que las secuencias blanco disponibles permiten su empleo para: 1) La estimación de diferentes parámetros genéticos en las poblaciones de las especies incluidas en este grupo, 2) Establecer relaciones de parentesco para identificación especie-específica y 3) Esclarecer el estatus taxonómico de las especies dentro del grupo Verrucarum.

Para la discusión de la información recopilada en ésta revisión, se tuvo en cuenta la cronología de uso del marcador molecular iniciando por el empleo de isoenzimas y continuando por el uso de marcadores cuyos polimorfismos son el resultado de la amplificación por PCR. 
Tabla 1. Marcadores moleculares para el análisis de la diversidad genética y filogenia de especies del género Lutzomyia (Grupo Verrucarum) *

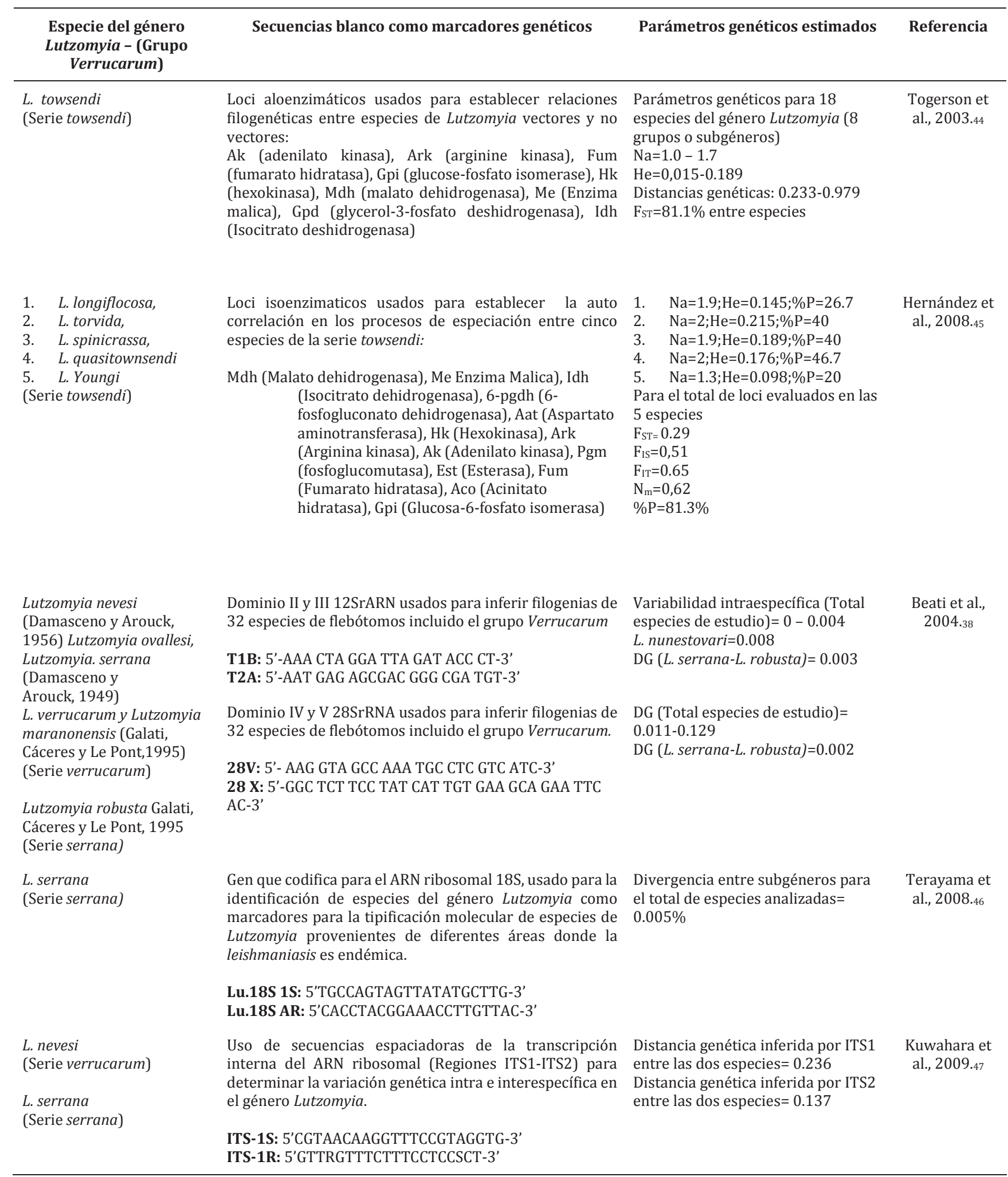




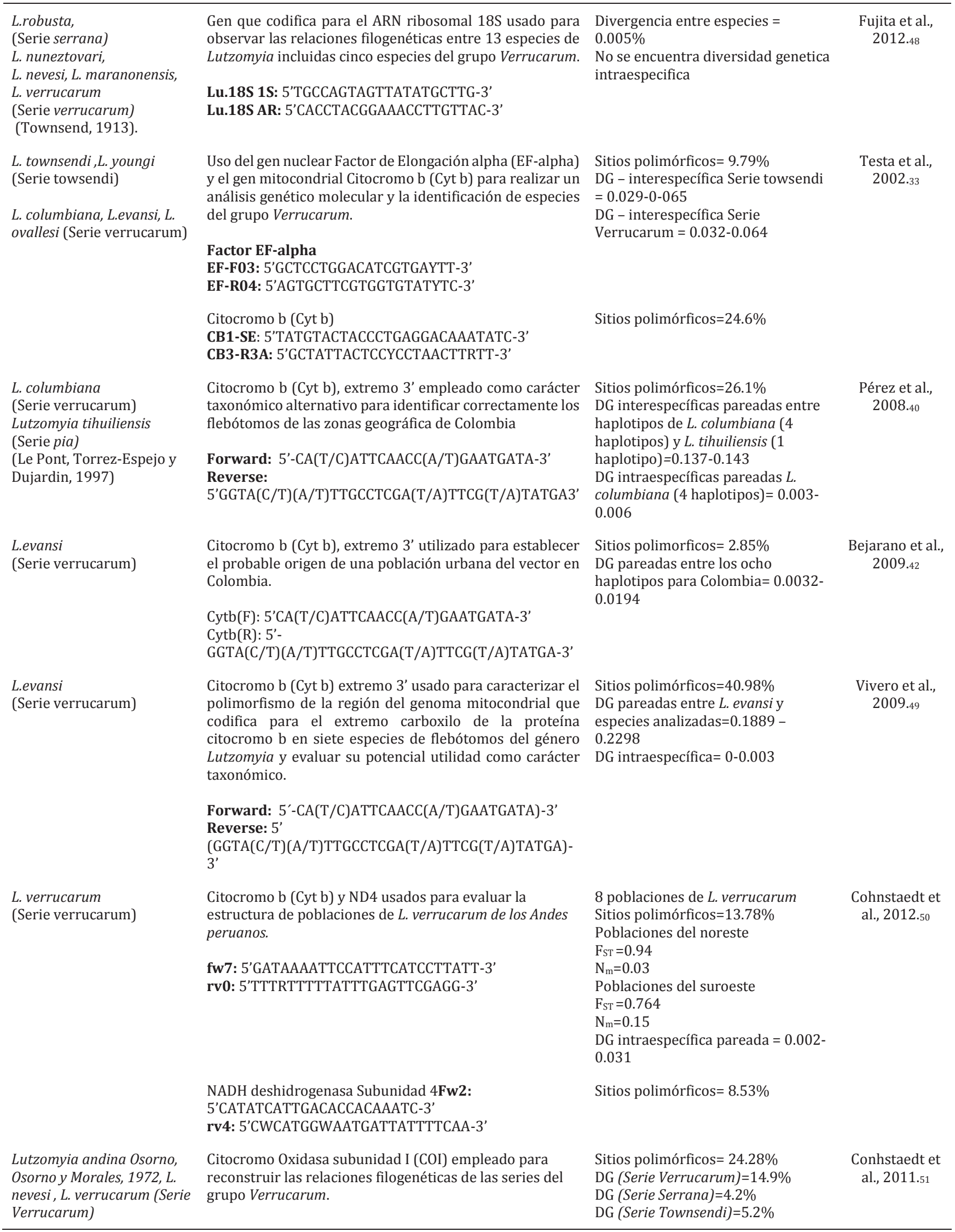




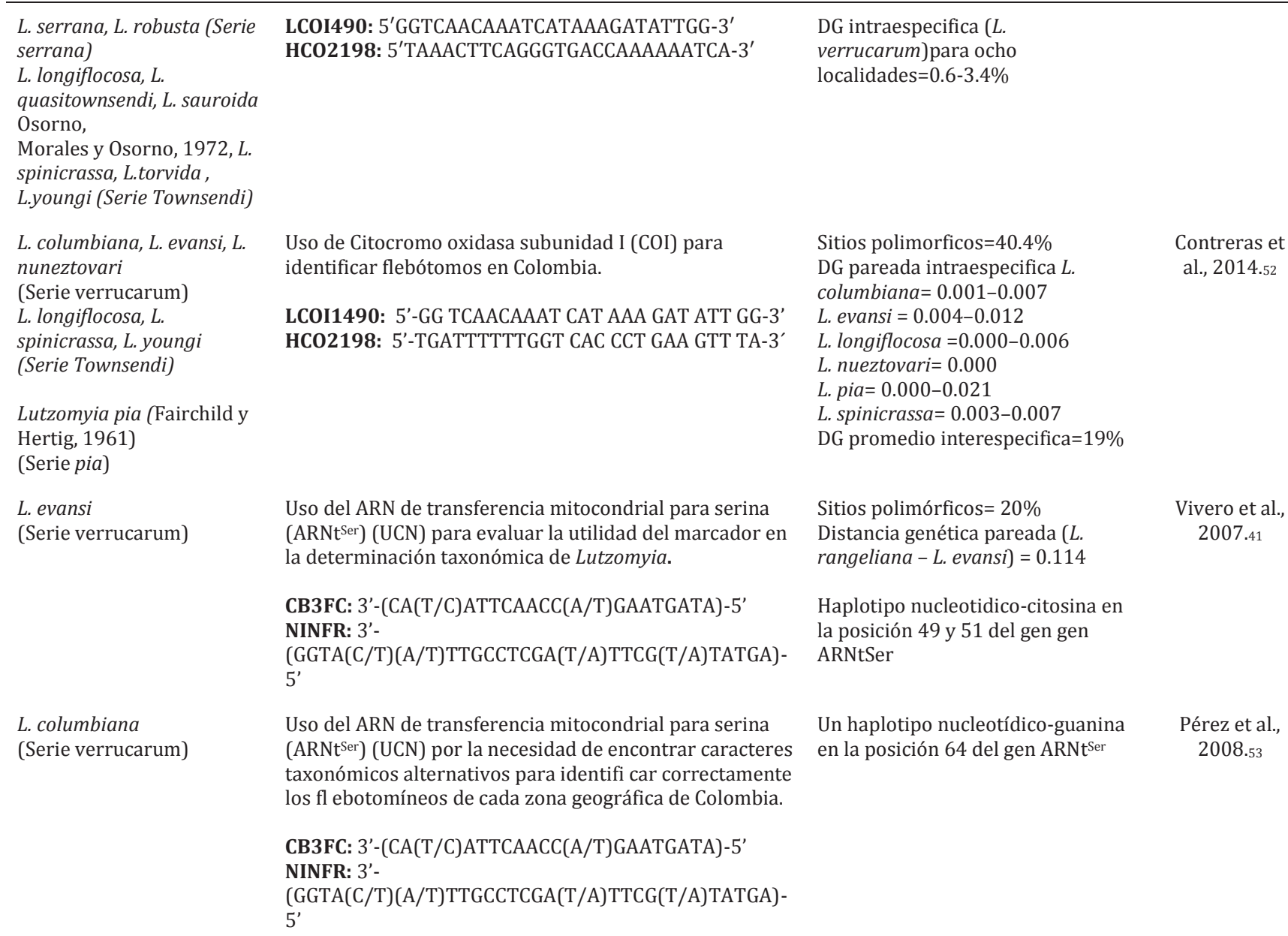

Na: Número de alelos privados; He: Heterocigosidad esperada; FST: Diferenciación genética entre poblaciones de una misma especie; FIT: Diferenciación genética entre individuos dentro de una población de una misma especie; $\mathrm{N}_{\mathrm{m}}$ : Número de migrantes efectivos; PI: Porcentaje de sitios polimórficos; DG: Distancia genética. \%P: Porcentaje de sitios polimórficos

*Nota: Secuencias blanco de los marcadores y valores de parámetros de diversidad genética obtenidos.

En el año 2003, Togerson et al.,44 analizaron en Venezuela la variación genética existente en 99 individuos empleando nueve loci aloenzimáticos, para siete subgéneros o grupos de especies dentro del género Lutzomyia y dos especies del género Brumptomyia. Se evidenció para el Grupo Verrucarum las especies L. ovallesi y L. townsendi.

De acuerdo con los datos de aloenzimas, las frecuencias de alelos variaron entre especies, algunos loci fueron compartidos por grupos de especies, mientras que otros loci fijaron diferencias entre especies distintas. Todas las poblaciones cumplieron con el equilibrio $\mathrm{H}-\mathrm{W}$, indicando apareamientos aleatorios dentro de ellas.39. Estos datos aloenzimáticos, mostraron niveles de variación genética entre los grupos morfológicos. Los grupos clásicamente adoptados por taxónomos de Lutzomyia representaron una proporción significativa de la variación genética entre especies porque las distancias genéticas obtenidas entre ejemplares de un grupo son menores que las obtenidas entre ejemplares de diferentes grupos. En consecuencia, los datos indican que los grupos de especies morfológicas hasta cierto punto, representan las relaciones genéticas entre especies.

En el análisis de los estadísticos $\mathrm{F}$, encontraron que el amplio componente de variación entre poblaciones - $\mathrm{F}_{\mathrm{ST}}$ es explicado por diferenciación entre especies $(81,1 \%)$ y un $19.9 \%$ adicional puede ser atribuida a diferencias entre grupos subgenéricos. Las distancias genéticas variaron 
de 0,233 a 0,979, siendo mayor entre $L$. rangeliana y L. shannoni (Grupo Psathyromyia) y menor entre L. venezuelensis y L. absonodonta, (Grupo Micropygomyia).44

En el año 2008, Hernández et al.,45 analizaron en 150 ejemplares procedentes de Colombia, 16 loci isoenzimáticos en cinco especies del grupo Verrucarum. Este marcador presentó un elevado polimorfismo para discriminar especies dentro del género Lutzomyia. El nivel medio de heterocigocidad esperada (He) fue bajo, atribuido principalmente a efectos fundadores recientes entre las localidades muestreadas o a un bajo flujo genético entre poblaciones en el seno de cada especie; al respecto, se observaron desviaciones del equilibrio Hardy-Weimberg (H-W) en 10 de los 16 loci, explicado por exceso de homocigotos, y atribuido principalmente a la ocurrencia diferencial de efecto Wahlund, subdivisión geográfica real y /o subdivisión temporal generacional. Igualmente, se determinó que hay una pronunciada diferenciación genética entre las especies, ratificando que son unidades reproductivas aisladas, reflejada posiblemente en la heterogeneidad entre las cinco especies, con ausencia de flujo genético entre las poblaciones.

De las cinco especies L. youngi fue la especie más divergente, con los niveles de heterocigosidad más bajos $(\mathrm{He}=0.098)$ y el menor número de alelos privados $(\mathrm{Na}=1.3)$, lo que soporta la hipótesis de la distribución de la especie a razón de la aparición del Istmo de Panamá permitiendo el paso entre Sur y Centroamérica. Los autores consideran que la clara diferenciación genética entre las especies $\left(\mathrm{F}_{\mathrm{ST}}=0.29\right)$, sin la presencia de híbridos, se explica por la selección natural dependiente de parches dispuestos al azar que por eventos estocásticos como la deriva genética.45

$\mathrm{Si}$ bien, las investigaciones con marcadores genéticos isoenzimáticos soportan en primera instancia la caracterización genética de las poblaciones, otros marcadores que usan la PCR también evidencian diferencias estadísticamente significativas entre las poblaciones de estos vectores de importancia para la salud pública. En la revisión realizada en 2010, por Kato et al.,54 los autores afirman que en los estudios basados solamente en los métodos de PCR-RFLP, se han caracterizado aproximadamente 400 especies de Lutzomyia y 100 especies de Phlebotomus, pero ha resultado imposible diferenciar todas las especies, aun cuando, usualmente, muchas de ellas coexistan en un área endémica determinada.

En el año 2000, Avise 5 describió las ventajas del ADN mitocondrial (ADNmt) como molécula estrella para el análisis filogenético; entre las características que favorecen su uso en la solución de problemas del conocimiento de las poblaciones y sus relaciones de parentesco se encuentran que es una molécula circular covalentemente cerrada, de tamaño pequeño, conformada por un total de 37 genes (13 ARN mensajeros, 2 ARN ribosomales y 22 de ARN de transferencia), además de una región conocida como región control o d-loop, encargada de controlar la transcripción y replicación en la molécula y tiene una tasa alta de substitución y de polimorfismo en muchos taxones.56

En el año 2004 Beati et al.,38 infirieron en Perú, filogenias de 32 especies de flebótomos que pertenecen a grupos de siete sub-géneros y dos especies, mediante el uso de fragmentos de la subunidad pequeña (12SrARN) y de las secuencias de la subunidad ribosomal grande (28SrARN). El subgénero Helcocyrtomyia y el grupo de especies del grupo Verrucarum estuvieron representados por 11 y 7 especies, respectivamente. Pese al limitado número de taxones, las filogenias resultantes, sobre la base de 837 caracteres, evidenciaron la base filogenética para la reconstrucción de las relaciones infragenéricas dentro Lutzomyia.

Terayama et al.,46 en 2008, compararon los patrones RFLPs del gen que codifica para el ARN ribosomal $18 \mathrm{~S}$ de especies de Lutzomyia en Ecuador, usando como enzimas de restricción AfaI y Hinfl. Los resultados evidenciaron la validez del marcador en la identificación especieespecífica, así como la estimación de parámetros de diversidad genética intraespecífica característica, con patrones únicos RFLP. Los autores confirman que la digestión con $A f a \mathrm{I}$ y subsecuente digestión con Hinfl de 18S rRNA son 
marcadores efectivos para la clasificación de las especies del nuevo mundo de Lutzomyia.

Un año después, Kuwahara et al.,47 utilizaron secuencias espaciadoras de la transcripción interna del ARN ribosomal (Regiones ITS1-ITS2) para estimar en Ecuador y Perú la estructura genética de poblaciones de Lutzomyia y Phlebotomus. El análisis de 44 individuos representados en 14 especies de Lutzomyia evidenció discrepancias entre la clasificación morfológica y las relaciones filogenéticas, encontrando que las dos especies del grupo Verrucarum se encuentran en grupos separados.

La distancia genética usando las secuencias de ITS1 dentro del grupo Verrucarum fue equivalente a 0,236 y mediante el marcador ITS2 fue menor e igual 0,13. Los resultados sugieren fuertemente que el marcador de ITS2 es un marcador más adecuado que ITS1 para el análisis taxonómico de especies de Lutzomyia incluyendo especies estrechamente relacionadas. Por otro lado, en el mismo estudio el uso de las secuencias ITS en el subgénero Helcocyrtomyia no evidenció una correlación entre la variación de las regiones y la distribución geográfica de las poblaciones de especies Lutzomyia hartmanni (Fairchild \& Hertig, 1957) y Lutzomyia ayacuchensis (Cáceres y Galati, 1988), cuyas poblaciones se encuentran en localidades con distancias superiores a 1200 $\mathrm{km}$, resultado que en la opinión de sus autores permite concluir que las secuencias ITS no son convenientes en estudios para estimar parámetros de estructura genética de las poblaciones de especies de Lutzomyia.

El uso de la técnica PCR-RFLP en la región que codifica para el gen de ARN ribosomal $18 \mathrm{~S}$ por Fujita et al.,48 en Perú en el año 2012, permitió la genotipificación de 1240 individuos agrupados en 13 especies de flebótomos, cinco de ellas pertenecientes al grupo Verrucarum. Los análisis de la región con las enzimas AfaI, HapII, KpnI y $X s p I$ permitieron diferenciar satisfactoriamente las especies de estudio. El análisis fue desarrollado principalmente para observar las relaciones filogenéticas entre las especies. Los resultados soportan la clasificación general basada en características morfológicas, excepto el agrupamiento entre las especies L. nuneztovari y L. verrucarum con especies del subgénero Helcocyrtomyia y Pifanomyia respectivamente, generándose una discrepancia en la clasificación. Fujita et al.,43 concluyen la necesidad de reconsiderar la clasificación de algunas especies de Lutzomyia por medio de análisis genómico, como el de secuencias del espacio de transcripción interno del ARN ribosomal y del gen citocromo b (Cyt b).

Es relevante anotar que el grupo Verrucarum incluye especies de Lutzomyia con hembras que además tienen una alta semejanza morfológica, es decir, que son consideradas especies crípticas o isomórficas, lo cual limita su identificación con las claves taxonómicas tradicionales. Testa et al.,33 en el año 2002 utilizaron secuencias de los genes Cyt b y factor de elongación alfa (EF-alpha) para establecer la identidad de miembros de la serie Townsendi, L. youngi (Venezuela) y L. townsendi (Colombia). Dentro del estudio, tres especies de la serie Verrucarum (L. columbiana, L. evansi y $L$. ovallesi) se seleccionaron como grupo externo para el análisis filogenético. Los resultados de filogenia molecular con las dos regiones (EFalpha y Cyt b) evidencian que la población de $L$. youngi de Venezuela es muy homogénea, y diferente a la población colombiana, resultando linajes diferentes, con un nivel probable de subespecies considerado por los autores del trabajo, y donde la existencia de haplotipos compartidos entre L. youngi de Colombia y $L$. townsendi colectada en Colombia, es explicada por introgresión.

Utilizando polimorfismo de conformación de cadena sencilla PCR (SSCP-PCR) 57 se han encontrado diferencias en las poblaciones de otras especies del género Lutzomyia como $L$. longipalpis utilizando análisis de haplotipos a partir de regiones mitocondriales de citocromo $b$. La variación en estos genes se ha demostrado útil en estudios de estructura genética y relaciones evolutivas entre taxa y puede ser contrastada con la ocurrencia de alopatría por la presencia de barreras geográficas y barreras climáticas como responsables del aislamiento y los patrones de migración.33,40,42,50,51,58 
Este fenómeno ha caracterizado la estructura de las poblaciones de los miembros del grupo Verrucarum y explicado principalmente por Alexander et al.,59 en 1992, quienes afirman que el rango de vuelo en el género Lutzomyia no excede los 100 metros en un periodo de 24 horas, lo que genera el aislamiento de la especie y en consecuencia la posible ocurrencia de deriva génica y/o presión por selección natural dependiendo del hábitat local, hecho que facilita a cada población desarrollar características.

Pérez et al.,40 en 2008 propusieron la necesidad de caracteres taxonómicos alternativos para identificar correctamente los flebótomos de cada zona geográfica de Colombia. Debido a la alta semejanza morfológica de algunas especies vectores con otras no vectores, secuenciaron el extremo 3' del gen mitocondrial que codifica para la proteína citocromo b en tres vectores potenciales de Leishmania presentes en el Valle de Aburrá, Lutzomyia hartmanni (Fairchild y Hertig, 1957), L. columbiana y L. tihuiliensis (. En el alineamiento múltiple de 321 nucleótidos del gen citocromo b de L. columbiana, L. hartmanni y $L$. tihuiliensis detectaron 83 sustituciones. En la secuencia parcial de la proteína encontraron 18 reemplazos de aminoácidos. Las distancias genéticas interespecíficas fluctuaron en un rango mínimo de 0,137 entre $L$. tihuiliensis y $L$. columbiana, y un máximo de 0,215 entre $L$. columbiana y L. hartmanni. Los polimorfismos detectados en la secuencia de nucleótidos del gen y de aminoácidos de la proteína constituyen caracteres moleculares potencialmente útiles para la determinación taxonómica de estas especies del género Lutzomyia.

En el estudio, Pérez et al.,40 reportaron que $L$. columbiana en el extremo 3' del gen homónimo tiene una longitud de 318 nucleótidos, que codifican para 105 aminoácidos y un codón de parada (UAA). A diferencia de esta especie, las secuencias homólogas de L. hartmanni y $L$. tihuiliensis codifican un aminoácido adicional, extendiéndose hasta el nucleótido 321. Según los autores, es probable que la ausencia de este aminoácido en L. columbiana corresponda al estado derivado de un carácter que evolucionó independientemente en diferentes linajes terminales luego de la separación del ancestro del grupo Verrucarum y el subgénero Helcocyrtomyia. Es importante señalar además, que en las secuencias de L. columbiana aparecieron dos codones de parada consecutivos; se plantea que en algunos animales este hecho puede estar relacionado con una reconfirmación de la señal de terminación durante la síntesis de algunas proteínas. Las distancias genéticas intraespecíficas oscilaron entre 0 y 0,006 para el caso de L. tihuiliensis y L. columbiana, respectivamente. Los valores inter-específicos fluctuaron en un rango mínimo de 0,137 entre L. tihuiliensis y $L$. columbiana, y un máximo de 0,215 entre $L$. columbiana y L. hartmanni. Esta última cifra supera ampliamente las distancias genéticas inter-específicas que se presentan al interior del grupo Verrucarum, mostrando una notable diferenciación subgenérica de L. hartmanni y concordante con la propuesta taxonómica de Young \& Duncan. En relación a la variación observada dentro de la especie L. columbiana, el marcador permite asociar individuos de la misma especie procedentes de regiones distantes, con una baja divergencia genética que oscila entre $0 \mathrm{y}$ 0.006.35 Este marcador molecular también permite discriminar especies dentro del grupo Verrucarum que presentan isometría e isomorfismo, como en el caso de L. tihuilienis con L. pia y que morfológicamente solo pueden diferenciarse por una variación en la pleura.60

Bejarano et al.,42 en 2009, utilizaron el gen mitocondrial citocromo b para establecer el probable origen de una población urbana del vector $L$. evansi en Colombia. El reporte que incluye 14 individuos de la especie L. evansi procedentes de seis zonas rurales y una zona urbana del Caribe colombiano, reveló una baja variabilidad genética entre zonas soportada por bajos valores de distancia genética $(<0,0194)$ e identidad genética entre los vectores procedentes de la zona rural y urbana. En el análisis genómico del gen se encontraron nueve sitios polimórficos, nueve haplotipos nucleotídicos y un solo haplotipo aminoacídico. Tres de los nueve haplotipos fueron compartidos entre individuos de la zona urbana y la zona rural, está última considerada como un foco de Leishmaniasis visceral desde 1980, hecho que posiblemente 
evidencia que la población urbana de L. evansi puede tener un origen por dispersión desde el foco rural de la enfermedad, teniendo en cuenta que estas localidades se encuentran a una distancia de $13 \mathrm{Km}$ y no hay barreras geográficas que puedan impedir tal dispersión.42

El extremo 3' del gen citocromo b también ha sido ampliamente empleado como un marcador eficiente en la identificación de especies. En Colombia, Vivero et al.,49 del Programa para el Estudio y Control de Enfermedades Tropicales PECET reportan en el año 2009, la presencia de polimorfismos genéticos en siete taxones del género Lutzomyia. El extremo carboxilo terminal de la proteína derivada de la misma evidencia además, sustituciones nucleotídicas, solapamiento de una o más bases con el gen tRNA ${ }^{\text {Ser }}$, reemplazo de aminoácidos, empleo de codones de parada distintos y diferencias en el tamaño del producto proteico. La sustitución nucleotídica de mayor trascendencia se observa en la posición 322 de L. rangeliana y L. evansi, con el cambio de guanina o adenina por timina, que conlleva a la disminución del tamaño de la proteína citocromo b codificada por estas especies. Se registran 29 sitios amino-acídicos polimórficos que generan un porcentaje de divergencia global del $26,6 \%$, útil para la discriminación de especie. Los sitios con mayor variación corresponden a las posiciones 39, 42 y 97 del alineamiento proteico. 49

Cohnstaedt et al.,50 en 2012, estudiaron genéticamente las poblaciones de $L$. verrucarum a partir del análisis del gen citocromo b y cuatro genes de la subunidad NADH deshidrogenasa en una población de 220 individuos procedentes de los Andes peruanos. Los genes de L. verrucarum se caracterizaron por fragmentos de 653 pb para el citocromo b y de 1125 pb para la subunidad NADH deshidrogenasa. La red de genes y análisis filogenéticos indicaron una alta similitud de haplotipos presentes en un solo valle $(0-0,52 \%$ divergencia de nucleótidos). Las moscas de cada valle tuvieron genotipos únicos no compartidos con muestras de otros valles o de regiones más distantes $(0,8$ a $3,1 \%$ de divergencia de nucleótidos). Así, los Andes del oriente conformados por tres haplotipos principales difieren de 12 a 13 pb y los Andes del occidente separados por $4 \mathrm{pb}$. Ahora bien, la estructura espacial sugiere importantes restricciones de movimiento entre los sistemas de valles, pero relativamente ninguna restricción por migración dentro de los valles. El alto grado de aislamiento y diferenciación genética entre los tres grupos (Fst $=0.944$ ) indican que las altas elevaciones de los Andes y los ambientes inhóspitos han sido efectivos en la prevención del flujo de genes entre las regiones geográficas $(\mathrm{Nm}=<0,18)$ entre valles y poblaciones separadas en unidades genéticas discretas. Según Cohnstaedt et al.,45 en los Andes las poblaciones de insectos podrían estar aisladas por distancias geográficas o barreras físicas creadas por disturbios geológicos. Por lo tanto, con la ausencia de pasos de baja elevación, la cadena de los Andes divide el hábitat de los insectos más que la separación por distancia en un ambiente de bosque tropical o un hábitat fragmentado por disturbios humanos.50

En la opinión de Cohnstaedt et al.,50 dentro de los valles individuales, la distribución de haplotipos y los insectos encontrados, formaron una continua población en entrecruzamiento. Los insectos pueden migrar solo cortas distancias generando una continua distribución del ADN mitocondrial a lo largo del valle, así las características geográficas, tales como la longitud del valle, y los cambios de elevación no impiden o dificultan la migración. Por otro lado, disturbios humanos tales como deforestación, cultivos, ciudades y carreteras no son barreras suficientes como para impedir el movimiento, pero estos disturbios pueden estar involucrados en cambios de efectos evolutivos en la estructura genética de las poblaciones. Se ha sugerido que, la destrucción del hábitat de los insectos vectores contribuye a la transmisión de enfermedades, debido a una rápida adaptación de las poblaciones de Lutzomyia a nuevos hábitats rurales y urbanos que favorecen su endofagia y antropofagia, al tener fuentes humanas y animales disponibles para su alimentación, situación que podría ayudar a la propagación de L. verrucarum entre y dentro de los Valles.50

En general hay concordancia entre las distancias genéticas y las relaciones filogenéticas dadas por 
los dos marcadores moleculares dando lugar a dos linajes principales. Un primer linaje que contiene todos los genotipos de los individuos colectados en la localidad tipo de Venezuela de $L$. youngi y de Mateguadua en Colombia y que de acuerdo con las características morfológicas de terminalia masculina corresponden a esta especie. Y un segundo linaje conteniendo todos los genotipos de todos los individuos colectados en Versalles y Jiguales en la Cordillera Occidental donde todos los machos tienen la morfología de $L$. townsendi y por ende, el linaje corresponde a esta especie.

Sin embargo, el análisis de secuencias con el gen Cyt b refleja la presencia de introgresión mitocondrial entre estas dos especies, explicado probablemente por hibridación interespecífica y en consecuencia generar una clasificación incompleta de un linaje. Esta introgresión es importante porque implica que rasgos vectoriales y asociaciones ecológicas ya no pueden ser vistos como propiedades fijas de diferentes morfoespecies. Adicionalmente, un resultado de gran importancia evidencia que, dos polimorfismos fijados e identificados en especies como L. youngi y L. townsendi pueden ser útiles como marcadores genéticos especie-específicos.

Otro de los genes mitocondriales reportado para la identificación y caracterización genética de vectores de leishmaniasis y bartonelosis es el gen citocromo c oxidasa I (COI). Recientemente, también se le atribuyen ventajas como marcador para examinar y comparar especies hermanas únicamente dentro del complejo L. longipalpis (Lutz y Neiva), así como, para reconstruir las relaciones filogenéticas entre series del grupo Verrucarum. 51

Cohnstaedt et al.,51 en 2011 reportaron para Perú y Colombia, el uso del gen COI como herramienta de identificación taxonómica entre las tres series de flebótomos que componen el grupo Verrucarum, series Serrana, Townsendi y Verrucarum.

Once especies de las tres series fueron incluidas en el estudio, procedentes de colecciones de los dos países; 1 la serie Verrucarum: $L$. verrucarum, $L$. andina (Osorno, Osorno-Mesa, y Morales), y $L$. nevesi (Damasceno y Arouck);2 La serie Serrana: L. serrana (Damasceno y Arouck) y L. robusta (Galati, Caceres, y LePont); y la serie Townsendi: L. longiflocosa (Osorno-Mesa, Morales, Osorno, y Muñoz de Hoyos), L. sauroida (Osorno-Mesa, Morales, y Osorno), L. torvida (Young, Morales, y Ferro), L. quasitownsendi (Osorno, Osorno-Mesa, y Morales), L. youngi (Feliciangeli y Murillo), y $L$. spinacrassa (Morales, Osorno-Mesa, Osorno, y Muñoz de Hoyos). Los 56 ejemplares de $L$. verrucarum fueron colectados de ocho localidades en el rango de distribución de la especie.

En el estudio se presenta una clasificación monofilética bien soportada para las tres series, indicando que el linaje basal de la serie Verrucarum probablemente diverge antes que las series Townsendi y Serrana; un 14,9\% de la divergencia genética es explicada entre tres especies dentro de la serie Verrucarum, mientras que $5.2 \%$ se explica entre seis especies de la serie Townsendi y sólo un $4.2 \%$ entre dos especies de la serie Serrana. ${ }_{51} \mathrm{Se}$ evidencia además, conespecificidad para cuatro especies, agrupadas en dos linajes parafiléticos, el par L. longiflocosa- $L$. sauroidea y el par L. quasitownsendi- Lu. Torvida; e inclusión de la especie L. nevesi en una serie distinta de Verrucarum, explicada por la alta divergencia interespecífica entre L. nevesi y el resto de especies de la Serie Verrucarum.43 Además, se evidencia la variación genética de $L$. verrucaria (Townsendi) en tres regiones geográficas de Perú: Amazónica, nororiente de los Andes y sur occidente de los Andes.

Los parámetros genéticos indicaron una variación intraespecífica de esta especie en las zonas y que difiere en un $0,6-3,4 \%$, encontrando similitud entre las poblaciones de L. Verrucarum del lado occidental de los Andes. De acuerdo con los autores, las poblaciones del nororiente de los Andes se caracterizan por una alta distancia genética con respecto al suroccidente, explicado por la limitada capacidad de dispersión de los flebótomos, conllevando a la generación de estructura genética por distancia y altos valores de diferenciación en presencia de barreras abióticas. En otras palabras, la heterogeneidad topográfica de los Andes combinado con la baja 
capacidad de dispersión, es un factor que limita la capacidad de las poblaciones para colonizar nuevas áreas y migrar entre sistemas de valles. En estas condiciones, la especiación alopátrica podría progresar rápidamente para dar lugar a individuos genéticamente distintos pero morfológicamente similares, la homogeneidad morfológica vista dentro de las series de especies puede ejemplificar este proceso.51

En el año 2014, Contreras et al.,52 reportaron el uso de citocromo oxidasa subunidad I (COI) como marcador específico para la asignación taxonómica de 148 individuos a especies, en 11 departamentos de Colombia. El análisis de 148 secuencias evidenció 103 haplotipos, identificando 36 (MOTUs) correspondientes a las 36 especies que han sido reconocidas por la taxonomía tradicional basada en caracteres morfológicos, de esta manera, haplotipos de una sola especie forman grupos de códigos de barra que son claramente distinguibles de otras especies relacionadas.

Por otro lado, los autores observaron divergencia intra-específica equivalente a $2 \%$ en la mayoría de los casos y divergencia interespecífica entre $9 \%$ a $26,7 \%$; estos resultados evidencian que el gen COI discrimina correctamente a individuos dentro de especies y a especies dentro de series como es el caso de L. spinicrassa, L. youngi y $L$. longiflocosa, tres especies clasificadas en la serie townsendi, consistente con la clasificación por caracteres morfológicos.52 Según los autores, la variabilidad intra-específica superior al $2 \%$, entre diferentes haplotipos de L. gomezi, L. panamensis y L. trinidadensis, sugiere probablemente una diferenciación poblacional o la presencia de especies cripticas; esta divergencia puede además, estar relacionada a la rápida variación del genoma mitocondrial o al rango y distribución de una especie que puede permitir el aislamiento y la diferenciación genética.

Los genes mitocondriales son una herramienta complementaria a la morfología en el estudio taxonómico de los flebotomíneos.58 Sin embargo, a la fecha solo se han analizado segmentos parciales de un número limitado de genes mitocondriales y falta aún explorar la utilidad taxonómica del resto del genoma mitocondrial que incluye los genes que codifican para los ARN de transferencia (tRNA) responsables de la síntesis de proteínas en la mitocondria.61

Los genes mitocondriales que codifican para los tRNAs se caracterizan por presentar una mayor tasa de mutación que los rRNA del genoma nuclear, los cuales son altamente conservados en insectos. Entre tanto, los genes que codifican para tRNAs mitocondriales evolucionan más lentamente que los genes mitocondriales codificadores de proteínas, razón por la cual podrían ser útiles como marcadores moleculares a distintos niveles taxonómicos.53

En insectos, los tRNAs se sintetizan inicialmente como moléculas monocatenarias de RNA, las cuales posteriormente experimentan apareamientos intracatenarios que dan origen a la estructura secundaria de doble cadena con forma de hoja de trébol. Sin embargo, estos genes están sometidos a restricciones mutacionales que operan especialmente en sus dominios funcionales, por consiguiente, la utilidad de los tRNAs estaría no solo en el polimorfismo de la secuencia primaria de nucleótidos, sino también en los cambios en la estructura secundaria de la molécula.53

Haciendo uso de estos marcadores, Vivero et al.,41 en 2007 realizaron un análisis del ARN de transferencia mitocondrial para serina (ARNtser), en 11 hembras de siete especies de Lutzomyia en el municipio de Sincelejo-Colombia. En cuanto a la secuencia primaria, el alineamiento indicó 14 sitios nucleotídicos polimórficos, incluyendo cuatro eventos indel (inserción-deleción), con una tasa de variación global de 20\%. En la estructura secundaria del gen ARNtser, la mayoría de los caracteres nucleotídicos variables se expresaron a lo largo de las lupas DHU, TyC y variable, así como al extremo basal del brazo anticodón, en los cuales se encontraron posiciones con nucleótidos propios de cada especie de flebótomos. Esto permite deducir que tales regiones presentan una mayor probabilidad de mutar en estos insectos. Para especies del grupo Verrucarum como Lu. evansi por ejemplo es exclusiva la presencia de la base citocina en los 
sitios 49 y 51 de la lupa TyC. Entre las secuencias nucleotídicas del gen mitocondrial ARNtser de las siete especies de Lutzomyia incluidas en el estudio, el $80 \%$ de la estructura esta conservada, posiblemente consecuencia de restricciones operacionales que hacen imperiosa la conservación de la estructura secundaria de este ARNt. Más aún, las regiones que aparecen estructuralmente conservadas, como el brazo aceptor del aminoácido, el brazo DHU, la lupa del anticodón y el brazo TyC, revelan la importancia funcional que tienen en el $\mathrm{ARNt}^{\mathrm{Ser}}$ de estos vectores de importancia para la salud.

Las sustituciones nucleotídicas en el gen ARNt ${ }^{\mathrm{Ser}} \mathrm{y}$ los rearreglos estructurales ocasionados por éstas, son útiles para la correcta distinción de cada uno de las especies de flebótomos estudiadas, sumado a las diferencias en el tamaño del gen por la pérdida o ganancia de bases.41 Estos cambios genéticos parecen soportar una tendencia evolutiva hacia la disminución del número de bases nucleotídicas $\mathrm{y}$, por consiguiente, en la disminución del tamaño de los elementos que integran el genoma mitocondrial. Hecho que puede explicar el solapamiento de la secuencia del gen ARNtSer con el gen citocromo b en L. cayennensis cayennensis, L. rangeliana y $L$. gomezi. 41

Pérez et al.,53 en 2008, describieron la estructura del ARN de transferencia mitocondrial para serina que reconoce el codón UCN (ARNtSer) en seis individuos del vector $L$. colombiana, en el departamento de Antioquia.4 En este estudio, el gen mitocondrial ARNtSer exhibió una longitud de 67 pares de bases (pb) y se encontró un solo haplotipo nucleotídico para el gen. Según sus autores, este resultado refleja la conservación evolutiva de esta molécula a nivel intraespecífico, denotando una baja dinámica mutacional y consecuencia de las complejas restricciones que operan el reconocimiento e interacción de la estructura tridimensional del ARNt ${ }^{S e r}$ y su función fisiológica. En la posición nucleotídica 64 del gen se observa la presencia de una guanina que distingue a L. columbiana del resto de las especies de flefótomos de América analizadas hasta ahora, las cuales presentan una adenina en ese lugar, y que produce un apareamiento no canónico tipo uracilo-guanina en el brazo aceptor de la estructura secundaria. Los autores evidencian además, que al comparar el gen $\mathrm{ARNt}^{\mathrm{Ser}}$ de ejemplares de L. columbiana de otras regiones como el Valle del Cauca y Envigado-Colombia, existe un porcentaje de similitud del $100 \%$, lo que demuestra la robustez del marcador mitocondrial para asociar individuos de la misma especie, pero procedentes de poblaciones ecológicas geográficamente distantes. De esta manera se considera entonces que este marcador molecular resulta ser importante en la caracterización genética y el diagnóstico de especie y por su patrón de evolución tARNser puede emplearse para la distinción de especies dentro del grupo Verrucarum.53

Montenegro et al.,62 en el año 2013, reportaron el registro de L. Columbiana como un flebótomo frecuente en focos históricos de bartonelosis en Colombia. Se sospecha que éste vector participó en la transmisión de la bacteria Bartonella baciliformis durante un brote de bartonelosis humana registrado en el departamento de Nariño-Colombia, que según los autores se observó en el sur del país entre los años 1935 y 1945 y generó la epidemia con mayor letalidad en éste departamento en las cuencas de los ríos Guáitara, Juanambú, Mayo y Patía. Este insecto ha sido también asociado con la transmisión del parásito Le. mexicana en un foco de leishmaniasis cutánea del suroccidente del país.3

Considerando el reporte realizado por Montenegro et al., 62 en el cual se evidencia para el año 2011 la dominancia de L. columbiana con porcentajes de abundancia relativa equivalentes al 98,45\% para el departamento de Nariño y la presencia confirmada de la especie en los municipios de Consacá, Samaniego y la UniónColombia, el Grupo Interdisciplinario de Investigación en Salud-Enfermedad de la Universidad Cooperativa de Colombia inició en el año 2013 un proyecto de investigación con el propósito de caracterizar molecularmente las poblaciones de este vector usando el gen ARTNtser (datos sin publicar).

Los resultados finales del estudio confirman la presencia del vector en cinco poblaciones del 
departamento de Nariño; Samaniego, Consacá, La Unión, San Pablo y San José. La clasificación taxonómica del estatus de especie se confirmó empleando la clave generada por Young y Duncan (1994) y Galati (2003). La clasificación taxonómica molecular fue confirmada a través de: 1) Alineamiento de 70 secuencias procedentes de ejemplares del departamento de NariñoColombia, colectadas en el 2014, con la secuencia reportada previamente 53 para L. columbiana en Colombia. 2) Obtención de un alineamiento del $100 \%$ de las secuencias y 3) Hallazgo de una secuencia con uno y dos sitios polimórficos respectivamente con respecto a la secuencia de referencia de ARNt de L. columbiana para las poblaciones San Pablo y San José.53 El uso del marcador del ARN de transferencia mitocondrial para serina que reconoce el codón UCN (ARNtSer) evidenció diversidades genéticas promedio diferentes a cero, y con valores de 0.084 para diversidad haplotípica y de 0.00026 para la diversidad nucleotídica.

\section{Conclusiones}

El uso de marcadores moleculares como herramienta de la taxonomía ha permitido determinar la identidad de los individuos de poblaciones naturales problemáticos para los criterios basados en caracteres morfológicos de la taxonomía clásica, facilitando la identificación a nivel específico. En adición, otros estudios, basados en parámetros de distancias genéticas, diferenciación entre pares de poblaciones y estimación del número de migrantes, han evidenciado en algunos casos, la presencia de poblaciones panmíticas y en otros una clara y marcada diferenciación de la estructura genética de las poblaciones involucradas.

Los resultados obtenidos en los estudios referenciados en esta revisión, reflejan los problemas en la clasificación de las especies del grupo Verrucarum. En consecuencia, los marcadores moleculares y en particular las secuencias de ADN, son ampliamente utilizados en la ciencia entomológica y son aplicados con frecuencia en la entomología médica. El uso de marcadores moleculares hace posible identificar haplotipos y postular la historia natural de las especies, aspectos de gran importancia en entomología médica.

Los datos moleculares posibilitan además, diferenciar especies simpátricas, alopátricas y parapátricas, así mismo, obtener estimativos relevantes para entender la dinámica y la estructura de las poblaciones naturales o estudiar aspectos como la resistencia a insecticidas y la relación parásito vector.

La taxonomía basada en caracteres morfológicos permite definir taxones a nivel genérico y específico, históricamente su legado es incuestionable y en la actualidad sus aportes en la definición de grupos naturales y en el área de la sistemática siguen vigentes. Los marcadores moleculares utilizados en la actualidad en biología molecular son una herramienta que define la identidad de grupos naturales problemáticos para la taxonomía clásica, que utiliza caracteres morfológicos basados en mediciones directas que reflejan su estado actual que en cierta medida desconoce un posible evento de plasticidad.

En consecuencia los marcadores moleculares ofrecen mediciones indirectas que representan los cambios acumulados en el genoma de un individuo, constituyéndose como herramientas contundentes en la resolución taxonómica de especies biológicas conflictivas y que en el área de salud pública es imprescindible realizar definiciones con celeridad. La revisión de los estudios realizados en el grupo Verrucarum, evidencian el uso frecuente de Loci aloenzimáticos, Dominio II y III 12SrARN, Dominio IV y V 28SrRNA, gen que codifica para el ARN ribosomal $18 \mathrm{~S}$ y citocromo oxidasa subunidad I (COI) como marcadores efectivos para inferir y reconstruir las relaciones filogenéticas entre especies o series del grupo Verrucarum y entre especies de flebótomos incluido éste grupo; mientras que el gen ARN ribosomal 18S, Factor de Elongación alpha (EFalpha), gen mitocondrial citocromo b (Cyt b) y ARN de transferencia mitocondrial para serina (ARNtSer) (UCN) se reportan como marcadores taxonómicos alternativos en la identificación de 
especies de flebótomos incluidos el grupo Verrucarum; por último el factor de elongación alpha (EF-alpha), el gen mitocondrial citocromo b (Cyt b) y NADH deshidrogenasa subunidad 4 (ND4) estuvieron asociados al uso en estudios para evaluar la estructura genética de poblaciones.

\section{Conflicto de intereses: Ninguno declarado por} los autores.

\section{Referencias}

1. Tesh R. The genus flevovirus and its vectors. Annu Rev Entomol. 1988; 33:169-81.

2. Anderson B, Neuman M. Bartonella spp. as emerging human pathogens. Clin Microbiol Rev. 1997; 10(2):20319.

3. Montoya J, Ferro C. Flebótomos (Díptera: Psychodidae) de Colombia. In: Amat G, Andrade M, Fernández F, editors. Insectos de Colombia. Bogotá; 1999. p. 211-45.

4. Gutiérrez, CM Hoyos L. Taxonomía, biología y morfología de flebotóminos. Manual para el estudio e identificación de vectores de leishmaniasis. 2009.

5. Theodor 0 . On the classification of American Phlebotominae. L Med Entomol. 1965; 2:171-197.

6. Young D, Duncan M. Guide to the identification and geographic distribution of Lutzomyia sand flies in México, the west indies, Central and South America (Diptera: Psychodidae). 1a ed. Publishers A, editor. Gainesville; 1994.

7. Galati E. Phylogenetic systematics of Phlebotominae (Diptera: Pychodidae) with emphasis on American groups. Bol Dir Malariol Saneam Amb 1995; 35:133-142.

8. Young DG. A review of the bloodsucking psychodid mea of Colombia (Díptera: Phlebotominae and Sycoracinae). Tech. Bull. 806, Agric. Exp. Station, IFAS, Univ. Florida, Gainesville.1979.

9. Feliciangeli MD. La fauna flebotómica (Diptera, Psychodidae) en Venezuela: Taxonomía y Distribución geográfica. Bol. Dir. Malariol. San. Amb. 1988; 28: 99113.

10. Cazorla D. Revisión del grupo Verucarum Theodor(1965)(Diptera, Psychodidae, Phlebotominae), Rev Ecol Latinoamer.1995;3:51-6.

11. Bejarano E, Rojas W, Uribe S, Vélez I. Sistemática de especies de Lutzomyia del grupo Verrucarum Theodor, 1965 (Diptera: Psychodidae). Biomédica. 2003; 23(1):87-102.

12. Kreutzer RD, Palau MT, Morales A, Ferro C, Feliciangeli D, Young DG. Genetics relationships among phlebotomine sand flies (Diptera: Psychodidae) in the Verrucarum species group. J Med Entomol 1990; 27: 18.

13. Feliciangeli MD, Murillo J. Lutzomyia youngi (Diptera: Psychodidae), a new phlebotomine sand fly previously misidentified as L. townsendi in endemic foci of cutaneous leishmaniasis in Venezuela and Costa Rica. J Med Entomol 1987; 24: 141-6.

14. Galati E. Phylogenetic systematics of Phlebotominae (Diptera, Psychodidae) with emphasis on American groups. Bol Dir Malariol y San Amb 1995; 35(Suppl.1):133- 42.

15. Le Pont F, Torrez-Espejo MJ, Dujardin JP. Phlébotomes de Bolivie: description de quatre nouvelles espèces de Lutzomyia (Diptera: Psychodidae). Ann Soc Entomol Fr 1997; 33: 55-64.

16. Speight $M$, Hunter M, Watt A. Ecology of insects: concepts and aplications. Londrés, Willey-Blackwell; 2010.

17. Arrivillaca J, Navarro JC, Felicungeli YM. Morfologia y quetotaxia de1 tagma cefhlico larval de Lutzomyia franqa 1924 (Diptera: Psychodidae): Proposición de un sistema de nomenclatura. Boletin de Entomologia Venezolana 1999; 14(1):1-13.

18. Galati EAB. Classificação de Phlebotominae. pp. 23-52. In, E. R. Rangel, R. Lainson (Organizersorgs.). Flebotomineos do Brasil. Editora Fiocruz, Rio de Janeiro, Brazil. 2003.

19. Ogusuku E, Perez JE. Head setae of the immature stages of Lutzomyia spp (Diptera: Psychodidae) fkom Perk Bol Malariol y San Amb 1995; 35 (1):257- 268.

20. Galati EAB, Cáceres AG, Le Pont F. Description of Lutzomyia (pifanomyia) robusta sp. (Diptera, Psychodidae, Phlebotominae) from Peruvian Equadorean interandean areas. Rev Saúde Pública 1995; 29: 89-99.

21. Pérez SP. Diferenciación morfométrica de las hembras de la serie townsendi del grupo Verrucarum, género Lutzomyia, presentes en Colombia en los focos endémicos de leishmaniasis cutánea (tesis). Santafé de Bogotá, Colombia: Universidad de La Salle; 1996.

22. Añez N, Valenta DT, Cazorla D, Quicke DJ, Feliciangeli MD. Multivariate analysis to discriminate species of phlebotomine sand flies (Diptera: Psychodidae): Lutzomyia townsendi, L. spinicrassa, and L. youngi. J Med Entomol 1997; 34:312-6.

23. Cazorla D, Yépez J, Trasmote A, Medina V. Análisis multivariante de la variación morfométrica infraespecífica de Lutzomyia evansi (Nuñez-Tovar, 1924) (Díptera: Phlebotominae) en focos endémicos de leishmaniasis del Estado Falcon, Venezuela. Resúmenes, XVI Congreso Venezolano de Entomología, Santa Ana de Coro, Venezuela; 1999:89.

24. Ogusuku E, Campos M, Pérez E. Variación estacional de las dimensiones alares de Lutzomyia verrucarum (Diptera: Psychodidae). Rev Per Ent 1991; 34:55.

25. Fausto AM, Feliciangeli MD, Maroli M, Mazzini M. Morphological study of the larval spiracular system in eight Lutzomyia species (Diptera: Psychodidae). Mem Inst Oswaldo Cruz 1998; 93:71-9.

26. Valenta DT, Anez N, Tang Y, Killick-Kendrick R. The genital atrium as a good taxonomic character to distinguish between species of phlebotomine sandflies (Diptera: Psychodidae) from Venezuela. Ann Trop Med Parasitol 1999; 93:389-99.

27. Feliciangeli MD, Castejon OC, Limongi J. Egg surface ultrastructure of eight New World phlebotomine sand 
fly species (Diptera: Psychodidae). J Med Entomol 1993; 30: 651-6.

28. Pérez JE, Ogusuku E. Chorion patterns on eggs of Lutzomyia sandflies from the Peruvian Andes. Med Vet Entomol 1997; 11:127-33.

29. Sierra D, Vélez ID, Uribe S. Identificación de Lutzomyia spp. (Diptera: Psychodidae) grupo Verrucarum por medio de microscopía electrónica de sus huevos. Rev Biol Trop 2000; 48:615-22.

30. Fausto AM, Feliciangeli MD, Maroli M, Mazzini M. Ootaxonomic investigation of five Lutzomyia species (Diptera, Psychodidae) from Venezuela. Mem Inst Oswaldo Cruz 2001; 96:197-204.

31. Young DG, Duncan MA. Guide to the identification and geographic distribution of Lutzomyia sand flies in Mexico, the West Indies, Central and South America (Diptera:Psychodidae). Mem Am Entomol. 1994; 54: 1881.

32. Galati EAB. Classificação morfologia e terminologia e identificação de adultos Vol. I. Apostila Disciplina HEP 5752. Bioecologia e Identificação de Phlebotominae. Departamento de Epidemiologia Faculdade de Saúde Pública Universidade de São Paulo Av. Dr. Arnaldo, 715, 01246-904, São Paulo, SP, Brasil. 2010.

33. Testa J, Montoya J, Cadena H, Oviedo M, Ready P. Molecular identification of vectors of Leishmania in Colombia: mitochondrial introgression in the Lutzomyia townsendi series. Acta Trop. 2002; 84 (3):205-18.

34. Rangel EF, Lainson R, Souza AA, Ready P, Azevedo ACR. Variation between geographical populations of Lutzomyia (Nyssomyia) whitmani (Antunes \& Coutinho, 1939) sensu lato (Diptera: Psychodidae: Phlebotominae) in Brazil. Mem Inst Oswaldo Cruz. 1996; 91: 43-50.

35. Dujardin, F. Pont, E. Bianchi Galati. Cryptic speciation suspected by morphometry within Lutzomyia runoides. Comptes Rendus de l'Académie des Sciences - Series III - Sciences de la Vie. 1999; 322(5): 375-382.

36. Uribe $\mathrm{S}$. The status of the Lutzomyia longipalpis species complex and possible implications for Leishmania transmission. Mem Inst Oswaldo Cruz. 1999; 94: 729 734.

37. Dedet JP, Desjeux P, and Derouin F. Ecologie d'un foyer de leishmaniose cutanee dans la region de Thies (Senegal, Afrique de l'Ouest. 4. Infestation spontanee et biologie de Phlebotomus duboscqi Neveu-Lemaire 1906. Bulletin de la Societe de Pathologie Exotique. 1982; 75: 588-589.

38. Beati L, Cáceres A, Lee J, Munstermann L. Systematic relationships among Lutzomyia sand flies (Diptera: Psychodidae) of Peru and Colombia based on the analysis of $12 \mathrm{~S}$ and $28 \mathrm{~S}$ ribosomal DNA sequences. Int J Parasitol. 2004; 34(1):225-34.

39. Lanteri AA., Loiácono MS, Margaría C. Aportes de la biología molecular a la conservación de los insectos. Pp.: 207-220. En: Costa, C., S.A. Vanin, J.M. Lobo \& A. Melic (eds.). Proyecto de Red Iberoamericana de Biogeografía y Entomología Sistemática PrIbes 2002. Sociedad Entomológica Aragonesa, m3m Monografías Tercer Milenio.

40. Pérez A, Bejarano E, Sierra D, Vélez I. Caracteres moleculares para la determinación taxonómica de tres especies de Lutzomyia (Diptera: Psychodidae), vectores potenciales de Leishmania presentes en el Valle de Aburrá, Colombia. Rev la Soc Entomológica Argentina. 2008; 67(3-4):99-108.

41. Vivero R, Contreras M, Bejarano E. Análisis de la estructura primaria y secundaria del RNA de transferencia mitocondrial para serina en siete especies de Lutzomyia. Biomédica. 2007; 27(3):429-38.

42. Bejarano EE, Rojas W, Uribe S, Vélez ID, Porter CH. Genetic analysis of a recently detected urban population of Lutzomyia evansi (Diptera: Psychodidae) in Colombia. Rev Soc Entomol Argent 2009; 68 (1-2): 1-7.

43. Cohnstaedt LW. Defining the ecology of the sand fly, Lutzomyia verrucarum (Diptera: Psychodidae) using population genetics and geographic information systems. Yale University 2009; 167.

44. Togerson D, Lampo M, Velasquez Y, Woo P. Genetic relationships among some species groups within the genus Lutzomyia (diptera: psychodidae). Am J Trop Med Hyg. 2003; 69(5):484-93.

45. Hernández C, Ruiz M, Munstermann L, Ferro C. Estructura genética en cinco especies de flebótomos (Lutzomyia spp.) de la serie townsendi, grupo Verrucarum, en Colombia (Diptera: Prychodidae). Rev Biol Trop. 2008; 56(4):1717-39.

46. Terayama Y, Kato H, Gómez E, Uezato H, Calvopiña M, Iwata $\mathrm{H}$, et al. Molecular typing of sand fly species (Diptera, Psychodidae, Phlebotominae) from areas endemic for Leishmaniasis in Ecuador by PCR-RFLP of $18 \mathrm{~S}$ ribosomal RNA gene. J Vet Med Sci. 2008;70(9):907-13.

47. Kuwahara $\mathrm{K}$, Kato $\mathrm{H}$, Gómez E, Uezato $\mathrm{H}$, Mimori $\mathrm{T}$, Yamamoto $\mathrm{Y}$, et al. Genetic diversity of ribosomal RNA internal transcribed spacer sequences in Lutzomyia species from areas endemic for new world cutaneous leishmaniasis. Acta Trop. 2009; 112:131-6.

48. Fujita M, Kato H, Caceres A, Gomez E, Velez L, Mimori T. Genotyping of sand fly species in Peruvian Andes where leishmaniasis is endemic. Act Trop. 2012; 121:93-8.

49. Vivero R, Contreras M, Bejarano E. Cambios en el extremo carboxilo terminal de citocromo b como carácter taxonómico en Lutzomyia (Diptera: Psychodidae). Rev Colomb Entomol. 2009; 35(1):83.

50. Cohnstaedt L, Caceres A, Beati L, Munsterman L. The population structure of Lutzomyia verrucarum (Diptera: Psycodidae), a Bartonella bacilliformis and Leishmania peruviana Vector in Peru. J Med Entomol 2012; 49(1):79-84.

51. Cohnstaedt L, Beati L, Caceres A, Ferro C, Munstermann Y. Phylogenetics of the phlebotomine sand fly group Verrucarum (Diptera: Psychodidae: Lutzomyia). Am J Trop Med Hyg. 2011; 84(6):913-22.

52. Contreras M. (Diptera, Psychodidae, Phlebotominae) in Colombia. PLoS One. 2014; 9(1):1-9.

53. Pérez A, Bejarano E, Vélez I. Descripción del ARN mitocondrial para serina (UCN) de Lutzomyia columbiana (Díptera, Psychodidae). Rev Bras Entomol. 2008; 52(4):591-4.

54. Kato H, Gomez E, Cáceres A, Uezato H, Mimori T, Hashiguchi Y. Molecular epidemiology for vector 
research on Leishmaniasis. Int J Environ Res Public Health. 2010; 7:814-26.

55. Avise J. Phylogeography: The history and formation of species. University H, editor. Cambridge; 2000.

56. Eguiarte L, Souza V, Aguirre X. Ecología molecular. Primera Ed. Marcó R, editor. Ciudad de Mexico; 2007.

57. Hodgkinson $H$, Birungi J, Haghpanah $M$, Joshi S, Munstermann L. Rapid identification of mitochondrial cytochrome b haplotypes by single strand conformation polymorphism in Lutzomyia longipalpis (Diptera: Psychodidae) populations Virginia. J Med Entomol. 2002;39(4):689-94.

58. Bejarano E. Nuevas herramientas para la clasificación taxonómica de los insectos vectores de Leishmaniosis: Utilidad de los genes mitocondriales. Biomédica. 2001; 21(2):182-91.

59. Alexander B, Ferro C, Young D, Morales A, Tesh R. Ecology of phlebotominesand flies (Diptera: Psychodidae) in a focus of Leishmania braziliensis in northeastern Colombia. Mem Inst Oswaldo Cruz. 1992; 87:387-95.

60. Pérez-Doria A, Bejarano EE Sierra D, Vélez ID. Molecular evidence confirms the taxonomic separation of Lutzomyia tihuiliensis from Lutzomyia pia (Diptera: Psychodidae) and the usefulness of pleural pigmentation patterns in species identification. Journal of Medical Entomology 2008; 45 (4): 653-659.

61. Pérez A, Bejarano E. tRNASer (UCN) Mitocondrial de Lutzomyia hartmanni predicción de la estructura secundaria del tRNASer (UCN) mitocondrial del flebotomíneo Lutzomyia hartmanni (Diptera: Psychodidae). Acta Biol Colomb. 2011; 16(1):87-94.

62. Montenegro F, Arroyo C, Vivero R, Uribe S. "Presencia del género Lutzomyia (Díptera: Psychodidae: Phlebotominae) en focos históricos de bartonelosis en el departamento de Nariño-Colombia."Rev Fac Salud Univ Surcolombiana. 2013; 5:33-6. 